\title{
ASPECTOS DEL DESARROLLO INSTITUCIONAL AMBIENTAL EN ARGENTINA
}

\author{
MARTA SUSANA JULIÁ ${ }^{1}$
}

Manizales, 2010-04-29 (Rev. 2010-05-25)

\section{RESUMEN}

Los problemas ambientales actuales demandan acciones y respuestas por parte de las instituciones que tienen a su cargo la ejecución de la política ambiental y que gestionan el ambiente en el territorio que les compete.

La dinámica propia de la temática ambiental ha impuesto un ritmo de respuesta institucional, política, administrativa y jurídica de tal naturaleza que la construcción teórica desde el derecho ambiental se ha visto, al menos, rezagada. Es en este sentido que, ante los escasos antecedentes en Argentina referidos a estudios jurídico-institucionales relacionados con la cuestión ambiental o ecológica entendida como problema político acuciante, nos propusimos intentar diferentes desarrollos teóricos y relevamientos de datos capaces de echar algo de luz a la situación en nuestro territorio.

En este trabajo precisaremos, en primer término, nociones como gestión pública del ambiente e institucionalidad ambiental. En segundo término, nos ocuparemos de aquello que denominamos "nuevo orden ambiental Nacional". En ese marco abordaremos lo que aparece como sus impactos directos en la sanción legislativa nacional y provincial, pero principalmente en las instituciones ambientales nacionales y de las diferentes jurisdicciones provinciales ${ }^{2}$. A los fines de este último punto, explicitaremos los diferentes modelos institucionales elaborados que nos permitieron caracterizar los organismos públicos estatales y su desarrollo. Por último, efectuamos algunas referencias a la trascendencia de la coordinación interjurisdiccional en materia ambiental.

Asimismo, cabe destacar que el presente se inscribe en el marco del desarrollo de una novedosa línea de investigación entre los estudios jurídico-ambientales en Argentina. La misma intenta abordar los problemas ecológicos desde el derecho pero sin descuidar la especificidad y complejidad de lo ambiental.

\section{PALABRAS CLAVE:}

Gestión pública, institucionalidad ambiental, derecho, administración ambiental, coordinación de políticas.

\section{ASPECTS OF THE INSTITUTIONAL ENVIRONMENTAL DEVELOPMENT IN ARGENTINA}

\section{ABSTRACT}

The current environmental problems demand action and answers from the institutions that are responsible for implementing the environmental policy and managing the environment in the territory of their competence. 
The typical dynamics of the environmental subject matter has set an institutional, political, administrative and legal answer rhythm of such nature that the theoretical construction from the Environmental Law has been, to say the least, left behind. It is in this sense that, in view of the scarce background referred to legal-institutional studies in Argentina in relation with the environmental or ecological matter -understood as an urgent political problem,- we decided to intend different theoretical developments and data surveys capable to throw a light upon this situation in our territory.

In this work we will specify, in first place, ideas such as public environmental management and environmental institutionalism. Secondly, we will see to what we call "new environmental National order." In this framework we will deal with what appears as its direct impact in the national and provincial legislative sanction, but mainly in the national environmental institutions and the different provincial jurisdictions2. For the purposes of this last point, we will explain the different institutional models developed which allowed us to characterize the state-owned public organisms and their development. Finally, we will make a reference to the importance of the interjurisdictional coordination in environmental matters.

By the same token, it is worth noting that this work is registered within the framework of an innovative research line in the Judicial-Environmental Studies in Argentina. It intends to deal with ecological problems from the Law but without neglecting the specificity and complexity of the environmental issues.

Key words: governance, environmental institutions, law, environmental management, policy coordination.

\section{LA GESTIÓN AMBIENTAL}

Si bien es evidente la existencia de la gestión ambiental gubernamental, si entendemos por tal la organización y desarrollo de un sector del Estado para atender estos problemas, es necesario precisar numerosos conceptos, establecer su alcance y significado jurídico, describirlos en el marco del sistema jurídico en que se han incorporado.

A través del uso y aplicación de los mencionados conceptos en el abordaje de situaciones jurídico-ambientales sometidos a la consideración de la administración pública y la administración de justicia, se ha incrementado el análisis doctrinario y jurisprudencial que también es importante tener en cuenta.

Plantear una visión de la gestión ambiental pública, como la que pretendemos abordar en el presente artículo, desde el derecho ambiental o desde una perspectiva ambiental del derecho nos impone describir un conjunto de conceptos para acordar en qué contexto de análisis hablamos de gestión ambiental, cuáles son los desafíos jurídicos actuales, qué rol asignamos a la coordinación de políticas para poner en consideración las sugerencias que realizamos.

Cuando nos referimos a la gestión ambiental gubernamental, tomamos la definición de Brañes que referido por Cancino (2003, p. 59) nos dice: "La gestión ambiental es considerada como el conjunto de actos normativos y materiales que buscan una ordenación del ambiente, que van desde la formulación de la política 
ambiental hasta la realización de acciones materiales que tienen ese propósito". "En la gestión ambiental se incluyen actos no sólo de las autoridades gubernamentales, sino también de la llamada sociedad civil, integrada por personas, grupos y organizaciones sociales y privadas" (Ibíd.).

Para describir la gestión, y siguiendo a Brañes, vamos a considerar los tres elementos que la componen: la política, el derecho y la administración ambientales.

La política ambiental se la define como un proceso de toma de decisiones respecto al ambiente deseado y posible, que una comunidad pretende en un lugar físico en una cultura determinada. Según Brañes (2001): "La política ambiental se entiende como el conjunto de las acciones que se diseñan para alcanzar una ordenación del medio ambiente".

Es por ello que cuando mencionamos a la gestión ambiental estamos haciendo referencia al contexto político jurídico e institucional en el que se desarrollan las acciones gubernamentales en un momento histórico determinado.

Compartimos la posición de Oszlak (1980) al referirse al estudio de la implementación de las políticas donde considera que:

también podría observarse que nuestro instrumental teórico es aún rudimentario para captar fenómenos esencialmente dinámicos como el de 'convertir' políticas en acción. Nuestros conceptos tienen un rango limitado de variabilidad, sea en contenido u horizonte temporal.

Más complejo aún es observar el proceso de conversión de las políticas ambientales en acciones, dado el horizonte temporal desde el inicio del estudio de esta problemática y el escaso tiempo transcurrido desde su inserción en el sector público.

La creación y puesta en marcha de nuevos espacios institucionales constituye la primera decisión de incorporar lo ambiental en la órbita gubernamental, y se establece tanto con la creación como con el desarrollo de los espacios institucionales ambientales.

Para analizar la gestión ambiental un primer elemento a considerar es la institución ambiental creada, que va a definir sus características y el espacio a ocupar en la gestión que se trate, otorgándole un perfil propio.

En las instituciones ambientales, por una parte son los aspectos administrativos los que van a darle contenido al espacio institucional, y por otra parte es la jerarquía otorgada la que determinará los recursos que se pretenden asignar y la entidad en el marco de la gestión que se trate.

Es por lo anteriormente destacado, que consideramos que la institucionalidad ambiental es un elemento importante en la definición de la gestión ambiental en los distintos niveles de Gobierno de los países.

Una vez institucionalizado el tema ambiental e incorporado en la estructura del Estado, se establece una nueva relación jurídica-ambiental entre el Estado y los administrados, lo que requiere el desarrollo de marcos normativos de diversas características, extensión y contenido. 
La misma institucionalización se realiza a través de normas de distinto tipo según nivel de la organización, entidad y característica del espacio creado (así se puede crear a través de una ley o decreto y su implementación por vía resolutiva).

El marco normativo deberá regular los instrumentos administrativos ambientales de tipo preventivo como: permisos, habilitaciones, certificados, evaluaciones de impacto ambiental, auditorias, entre otros. A estos instrumentos pueden someterse las nuevas actividades que se desarrollan y que requieren de autorización por parte del Estado o que están sometidas a ciertas pautas ambientales.

Creado el espacio, institucionalizado y regulado da lugar a la tarea propia del sector que conforma lo que denominamos "administración ambiental", la que incluye las distintas responsabilidades que tiene a cargo tales como: implementar las políticas ambientales fijadas, cumplir los objetivos, desarrollar programas y acciones, fiscalizar, controlar y realizar todas las actividades que como autoridad de aplicación de las leyes le correspondan.

La administración ambiental reúne toda la actividad fiscalizadora y de control por parte de los organismos públicos existentes y en aquellos que pueden crearse o ampliarse en materia ambiental, de acuerdo a las facultades que otorgan las leyes en el marco de cada jurisdicción.

Por lo que la actividad de la administración requiere asociarse también a la generación de procedimientos, recursos, y modalidades de relación formal entre los administrados y el Estado en sus distintos ámbitos.

Establecida la gestión ambiental gubernamental produce un impacto directo en el derecho, ya que demanda la implementación de la política y el diseño del marco normativo necesario para que la gestión pueda llevarse a cabo.

También dicho impacto puede observarse en el ámbito privado, con relación al cumplimiento de los marcos normativos, en su fase sólo de cumplimentar las nuevas normas y también en los procesos voluntarios, ya que son verificados por los responsables que desarrollan actividades en el territorio que les va a permitir certificar sus procesos en tanto cumplan con la normativa vigente.

A partir del incremento de la gestión ambiental pública se produce un importante impulso en la producción legislativa, en las actividades de control, en la verificación de cumplimiento en el ámbito de desarrollo de la misma.

Un tema no menos importante es la participación ciudadana en la toma de decisión sobre temas que impactan en materia ambiental, para lo cual ha sido necesario crear las vías o instancias a través de las cuales los ciudadanos participen. Las áreas ambientales de las distintas administraciones en Argentina han creado vías o canales de participación para obtener información del Estado o para participar en las decisiones que afectan a los ciudadanos. Así por ejemplo la creación de consejos honorarios, las vías para denuncias ambientales, teléfonos verdes, las audiencias públicas, son distintas manifestaciones tanto formales como informales para acceder a la información y participar en el tema.

Como complemento de lo que hasta aquí destacado, la incorporación de la dimensión ambiental dio origen al desarrollo de vías formales de resolución de 
conflictos ambientales tanto administrativos como judiciales, cada una con sus procedimientos y particularidades.

En el contexto de la gestión ambiental gubernamental en Argentina, y considerando los aspectos que destacamos, queremos profundizar en los nuevos desafíos que enfrenta la gestión teniendo en cuenta los requerimientos de coordinación de las políticas públicas. En el contexto de la gestión ambiental gubernamental en Argentina, a mediados de los 90 surge un nuevo orden ambiental Nacional a partir de la reforma constitucional de 1994 que jerarquizó al más alto nivel normativo la cuestión ambiental.

\section{EL NUEVO ORDEN AMBIENTAL NACIONAL}

La incorporación de normas ambientales como leyes generales o leyes orgánicas, constituyó la configuración inicial de la normativa propiamente ambiental. Si bien en sus comienzos se establecieron principios y prohibiciones generales, de carácter declarativo, con pocas sanciones, en su evolución se van complementando a través del tiempo.

Algunos países desarrollaron sus normas generales u orgánicas en materia ambiental y sus normativas específicas, en algunos casos sin contar con la incorporación a nivel constitucional.

El derecho ambiental se va conformando con una mixtura entre lo viejo y lo nuevo, la interrelación en los distintos sistemas jurídicos de las normas de relevancia ambiental, propiamente ambientales y las de relevancia casual, de acuerdo a la clasificación que realiza Brañes (2001) sobre la legislación ambiental.

El derecho ambiental se manifiesta en la legislación ambiental, y se lo puede definir como el conjunto de instrumentos legales que forman parte de la regulación de la problemática ambiental. En una caracterización de la legislación ambiental argentina se puede decir que es numerosa, que proviene de épocas diferentes, dictada con diversos objetivos y donde a partir de una legislación difusa se fue construyendo el orden jurídico ambiental.

En Argentina, las últimas décadas delinearon y otorgaron un perfil jurídico, institucional y administrativo a las gestiones ambientales en los distintos niveles de Gobierno, avanzando en numerosos aspectos desde las provincias y municipios hasta el reconocimiento constitucional con la reforma de 1994 a la Constitución Nacional.

En el sistema argentino la incorporación de la cláusula ambiental en la Constitución por una parte, y el dictado de las leyes de presupuestos mínimos ambientales por otra, han modificado el esquema jurídico-ambiental existente configurando un nuevo orden ambiental a nivel nacional.

Las provincias y municipios en sus ámbitos y competencias han generado su legislación ambiental propia, la que deben integrar, adecuar y complementar a la de presupuestos mínimos nacionales y al mandato constitucional. 
La incorporación de la cláusula ambiental en la Constitución en la reforma de 1994, se establece en el capítulo sobre "nuevos derechos" y particularmente en el art. 41, incorporando un concepto amplio de ambiente. ${ }^{3}$

El análisis del artículo 41 requiere puntualizar distintos aspectos de su formulación, tratando de seguir el orden establecido en el mismo, destacamos en el primer párrafo la formulación del derecho al ambiente con un criterio amplio desde el punto de vista de su definición.

El concepto de ambiente, su alcance y significado, es relevante en tanto va a demarcar los límites en la interpretación de aquello que debe considerarse ambiental y que no. Las posturas al respecto dan lugar a tres enfoques sobre el derecho al ambiente: restringido, amplio y social de acuerdo a la interpretación desarrollada por Etchichury (2006, p. 20 y s.s.) con precisión.

Es el concepto mismo de ambiente en el sistema el que va a establecer dónde empiezan y terminan los problemas ambientales, condicionando de esta forma toda tarea posterior en materia jurídico, político e institucional.

El concepto de ambiente va a tener una trascendencia a la hora de interpretar y ubicar la temática ambiental tanto en el sistema jurídico, como en su aplicación en la gestión.

Las autoridades deben gestionar para proteger, pero no sólo se limita a ello sino que se refiere a qué debe tener en cuenta en la gestión: esto es al uso racional de los recursos, la preservación del patrimonio natural y cultural, la diversidad biológica y la información y educación ambientales, confirmando el criterio amplio de interpretación que debe seguirse en el tema.

Este proveer por parte de las autoridades constituye un verdadero imperativo y se relaciona estrechamente con la gestión, ya que hace hincapié en un conjunto de actividades a tener en cuenta en el desarrollo de la gestiones de cada jurisdicción.

El tercer párrafo es probablemente el más conocido y debatido del artículo, ya que establece la distribución de competencias en materia ambiental en el sistema e introduce la facultad de la Nación de dictar los presupuestos mínimos de protección ambiental.

Existe una importante discusión en torno al concepto , significado y alcance del término 'presupuestos mínimos' y a su vez las modalidades a tener en cuenta en las leyes.

El artículo 41, además de las referencias realizadas, ha modificado sustancialmente muchos elementos del sistema jurídico, operando cambios en distintos aspectos normativos, lo que implica un efecto cascada en un conjunto de temas asociados del sistema.

\section{LA LEY GENERAL DEL AMBIENTE, EL GOBIERNO NACIONAL Y LA GESTIÓN AMBIENTAL}

La facultad que otorga a la Nación el art. 41 de dictar las normas de presupuestos mínimos de protección ambiental recién se vio materializada a 
partir de 2002 donde aparecen las primeras leyes de este tipo, hoy seis vigentes, todas sancionadas entre 2002-2003.

Su inserción en el sistema no fue pacífica, generando discusiones en cuanto a su contenido, su constitucionalidad en algunos casos, las modificaciones que provocan, dando lugar a distintas posiciones doctrinarias, de técnicos y especialistas sobre el concepto de presupuestos mínimos y las leyes vigentes.

Una de las leyes sancionadas bajo esta modalidad es la Ley 25.675, Ley General del Ambiente, dictada como ley de presupuestos mínimos que viene a completar el nuevo orden ambiental establecido en el art. 41 de la Constitución.

Creemos que significa un nuevo orden por que importa el cumplimiento de lo que textualmente establece en el art. 3:

La presente ley regirá en todo el territorio de la Nación, sus disposiciones son de orden público, se utilizarán para la interpretación y aplicación de la legislación específica sobre la materia, la cual mantendrá su vigencia en cuanto no se oponga a los principios y disposiciones contenidas en ésta.

La Ley General presenta entre otras particularidades los objetivos de la política ambiental, los instrumentos de la gestión ambiental y tal vez lo más relevante es el reconocimiento de un conjunto de principios ambientales, tanto como la declaración de orden público de la norma.

Nos interesa destacar los objetivos que establece la ley que debe cumplir la política nacional, que por primera vez se explicitan en una norma legal en Argentina como tales. ${ }^{4}$

Esta determinación de los objetivos de política, sobre los que no vamos a profundizar, se impone con la sanción de la Ley General en 2003, de esta forma nos va a permitir en el tiempo considerar el cumplimiento o no de los objetivos planteados en el desarrollo de la política ambiental nacional.

Cómo se observa la gestión ambiental nacional y cuáles son los indicadores más relevantes en la actualidad teniendo en cuenta la conjunción de sus elementos: política, derecho y administración.

La existencia de un conjunto de objetivos determinados por la Ley General del Ambiente que se complementan con las obligaciones y deberes establecidos en la propia Constitución en el art. 41, conforman el marco normativo desde el cual se va a delinear la gestión ambiental nacional.

El desarrollo institucional ambiental desde su organización inicial (hace más de tres décadas) hasta su constitución actual, ha conformado con el tiempo un espacio que ha crecido en entidad hasta constituirse en una política de Estado. Así lo ha explicitado el ex Presidente de la Nación, Néstor Kirchner, en discurso efectuado el 5 de mayo del 2006 en la ciudad de Gualeguaychú, ante el conflicto por Botnia en la provincia de Entre Ríos:

En la región y en nuestro país debemos incorporar la dimensión ambiental en todos los niveles de Gobierno, optimizando el uso de instrumentos tales como el ordenamiento territorial, la obligatoria evaluación del impacto ambiental, la adopción de sistemas de diagnóstico e información ambiental, la participación ciudadana y el régimen económico de desarrollo sostenible. 
La protección de un ambiente sano debe estar en el centro de nuestras políticas de Estado, en el marco de una verdadera responsabilidad intergeneracional. ${ }^{5}$

La referencia del ex Presidente es quizá el hecho más significativo, en cuanto a política ambiental se refiere, al anunciar la decisión de convertirla en una política de Estado situación que la temática ambiental no tuvo con anterioridad.

La jerarquización de la Secretaría de Ambiente y Desarrollo Sustentable instalándola en la Jefatura de Gabinete de Ministros (Decreto 830/2006) constituye otro de los indicadores del espacio asignado a la gestión, ya que se incorpora en un área netamente de coordinación.

Como todo esto ocurre en el último año de gestión de un Gobierno, no es posible evaluar las asignaciones de personal y presupuestaria y los avances administrativos sin una lectura absolutamente parcializada. La gestión del Gobierno 2003-2007 es el marco adecuado para el mencionado análisis, que no es el objeto del presente artículo sino sólo la referencia general de la situación para plantear los desafíos a que se enfrentan las gestiones y con qué cuentan para ello.

Si se mantiene el espacio y la jerarquía, probablemente se convierta en una gestión ambiental nacional a estudiar en cuanto a la implementación de todos los aspectos declamados, observando cómo se manifiestan en el territorio las acciones proyectadas.

La administración ambiental es el aspecto más complejo, ya que el inicio de gestiones en áreas nuevas comprende la inserción administrativa en la estructura que se trate, su implementación responde a aspectos externos e internos a la propia área.

A ello debe agregarse en el caso de Argentina, la competencia concurrente de la temática ambiental otorgada en la Constitución y la competencia de las provincias en cuanto al dominio originario que tienen sobre sus recursos naturales.

La complejidad de competencias y jurisdicciones, es un elemento que por una parte limita la gestión ambiental nacional en tanto existen gestiones ambientales provinciales y municipales con competencia; por otra parte, concurren las competencias en ciertas materias y situaciones, se excluye la competencia local en las situaciones de interjurisdiccionalidad de ciertas situaciones ambientales.

La administración ambiental nacional tiene un rol central, que es la coordinación política e institucional con las distintas jurisdicciones involucradas en las temáticas que interviene.

Un elemento importante es el derecho como parte de la gestión, se ha desarrollado rápidamente respondiendo a las exigencias políticas e institucionales de cada organismo generando nuevas leyes, decretos, resoluciones siendo el vehículo para la implementación de muchos aspectos de la gestión.

Si bien el derecho ha pasado de una instancia inicial meramente declarativa al desarrollo de su faz administrativa y pública, con buenos desarrollos en las áreas de prevención tiene un importante camino para recorrer como elemento de la gestión ambiental. 


\section{LOS MODELOS INSTITUCIONALES, CARACTERIZACIÓN, TIPOS}

El análisis de la inserción de la dimensión ambiental en la gestión pública y los modelos de institucionalización ensayados en Argentina, nos conduce a reflexionar sobre un conjunto de aspectos asociados a la institucionalidad y la formulación de políticas ambientales en ese contexto.

Nos preguntamos acerca de los principales modelos institucionales ensayados, tratando de encontrar ciertas particularidades que nos permitan caracterizarlos, lo que nos permitió llegar a la siguiente sistematización teniendo en cuenta los criterios tomados en cuenta:

a- Modelo netamente ambiental de mayor jerarquía, comprende la creación de ministerios y secretarías de Estado en la temática ambiental. b- Modelos con menor jerarquía y dependencia funcional de ministerios de objeto compartido. c- Modelos con menor jerarquía y dependencia funcional de ministerios con otra temática.

d- Modelo no estructurado en organismos tradicionales.

De acuerdo a las distintas formas de institucionalización ambiental en Argentina, a través del tiempo se han elaborado cuatro categorías de modelos institucionales.

Ello nos permite sistematizar las instituciones existentes en estas categorías ubicando su jerarquía en la administración de cada jurisdicción en función de su dependencia, indicando su cercanía al Ejecutivo, el modelo de mayor jerarquía que puede competir con los modelos estructurados tradicionalmente que dependerán de su autarquía y relación con el Ejecutivo.

Cada modelo representa un conjunto de condiciones que permiten desarrollar la institución de acuerdo al espacio, jerarquía y trascendencia otorgada en la gestión que se analiza.

¿La elección de un modelo, condiciona el tipo de institucionalidad? Nos preguntamos cómo y de qué manera se construye la institucionalidad ambiental en

Argentina y qué factores contribuyen o no al desarrollo de la misma.

Se inserta un espacio en la estructura de Gobierno, se selecciona un modelo de institución, comienza un proceso de construcción de la institucionalidad ambiental. Todo ello, constituye una decisión política del jefe de la gestión gubernamental acerca del lugar, espacio, imagen o visibilidad social de la temática en su Gobierno.

Institucionalizada el área ambiental por decisión política del gestor o por el legislador a través de las leyes generales que requieran una autoridad de aplicación ambiental, se construye el área ambiental.

Ante la institucionalización de la problemática en una gestión aparecen percepciones internas y externas a la gestión pública. 
Cada modelo va a generar percepciones internas sobre espacio, jerarquía, competencia, atribuciones, facultades y alcance con relación al sector desde el que se observa. Por ejemplo, con áreas más cercanas en la gestión de los problemas se manifestarán en conflictos o zonas grises para abordar, coordinar o deslindar competencias temáticas, instrumentales, de fiscalización y control. Por el contrario, con áreas más lejanas a las cuales son extrañas serán percibidas por la curiosidad de la problemática.

La percepción externa de la institucionalidad, de acuerdo a los modelos, será observada desde las distintas organizaciones privadas y la sociedad civil generando mayor demanda de acuerdo al grado de confianza en la posibilidad de gestionar problemas o de simple indiferencia.

Instalar un modelo

Entre otras cosas, instalar un modelo implica generar percepción externa e interna; describimos algunos rasgos de acuerdo al modelo seleccionado:

a) Los organismos netamente ambientales de alta jerarquía como ministerios o secretarías de Estado aparecen, en sí mismos, como una asignación de importancia al tema en una gestión política determinada.

Instalar un ministerio o secretaría de Estado cobra significado, hace visible y ubica el tema en agenda por la envergadura y entidad del espacio.

El impacto de la construcción de la institución o su jerarquización, se observa en la inclusión de nuevos funcionarios en el gabinete con otra voz en las reuniones y discusiones temáticas.

La elección de mayor jerarquía y espacio tiene como contrapartida compromiso y presencia de una temática compleja en la gestión. El tipo de modelo institucional seleccionado va a estar condicionado por la asignación de recursos, humanos y materiales, capacitación técnica del personal que permita asumir el rol asignado. Crear instituciones, elegir modelos y desarrollarlos demanda la formulación de políticas ambientales en su fase más ejecutiva y específica como son los programas o acciones de los organismos.

Las instituciones ambientales ejecutan políticas en el territorio con los instrumentos de gestión con que cuenten: ordenamiento territorial, E.I.A., control y fiscalización, educación, información, para citar algunos de ellos.

Los marcos normativos claros permiten identificar los objetivos definidos para la problemática, de allí la importancia que tienen las leyes generales al establecer no sólo los objetivos sino muchos de los instrumentos de política y gestión del ambiente.

b) Los modelos de áreas compartidas

La principal diferencia entre un área netamente ambiental como la descripta anteriormente y una de objeto compartido, es la competencia y deferencias en las prioridades con el área que se vincula.

Las relaciones históricas, rivalidades, conflictos o tensiones entre las temáticas que se comparten y la fase operativa generan actuaciones diferentes y problemas en el abordaje de las situaciones a enfrentar. 
Las prioridades ante la tensión o conflicto entre dos temáticas como por ejemplo: ambiente y producción o la consideración del uso y explotación de un recurso y el estado de situación del ambiente en que se encuentra, u otros casos donde el uso y administración del recurso entra en conflicto con la calidad del mismo.

Esto suponte la toma de decisión en cuanto a la valorización de las problemáticas, aplicación de la tutela que contienen las normas que la regulan y análisis de las jerarquías frente a las tensiones o conflictos en los contextos más amplios desde los cuales comprenderlos.

Objetos compartidos supone integralidad en la gestión de los problemas, pero no generar desequilibrios o inequidades en el tratamiento de las situaciones por parte de las instituciones.

\section{c) Los modelos de baja jerarquía e institucionalidad}

La adopción de modelos que han institucionalizado la temática ambiental con escasa jerarquía y espacio, trae como primera consecuencia el estar excluidos de la discusión política o de la participación del tema en las discusiones de la agenda política, o dicho de otro modo la no injerencia en las discusiones en el ámbito del gabinete.

En materia de política no es un tema menor, ya que el tratamiento de los problemas generales de Gobierno no incluirá la dimensión ambiental con la fuerza de la presencia del funcionario que entiende en el tema y que enfrenta los problemas.

Esta ausencia en la decisión política le va a traer aparejado otras limitaciones como las presupuestarias y de recursos en general.

Se torna en un área poco visible en el contexto de la gestión pública, y por tanto en la percepción interna que tienen el resto de los organismos de la gestión.

Alcanzar recursos, capacitación y formulación de políticas específicas es mucho más difícil en este tipo de modelos. Los programas y acciones vienen delineados desde las áreas donde se encuentran insertos, cuyos objetivos pueden entrar en contradicción con los del organismo ambiental.

Constituir la autoridad de aplicación de las leyes ambientales, se convierte en un contrapeso por el control y fiscalización en condiciones limitadas de institucionalidad.

d) Los modelos no tradicionales

Se instalan modelos no tradicionales con instituciones nuevas que tienen que demostrar y ganar el espacio en la gestión. Suelen generar desconfianza frente al cambio o a la nueva o poco conocida modalidad de gestión de la temática.

En algunas gestiones estos organismos son marginados por el resto de los organismos. Las áreas deben desarrollar un proceso propio, no realizado con anterioridad, de tal manera de lograr un lugar con entidad suficiente en la gestión que se inserta. 
Tienen una doble construcción de su imagen hacia fuera y hacia dentro de la gestión pública.

La percepción inicial de las nuevas figuras es de desconfianza hasta que se difunde y conoce su existencia como maneras de accionar, la que acercará en más o en menos a los sectores involucrados en la temática.

\section{LAS PROVINCIAS Y SUS OBLIGACIONES AMBIENTALES}

El desarrollo de la gestión ambiental en las provincias ha sido variado y de acuerdo a sus particularidades geográficas, climáticas, y condicionadas por los problemas ambientales centrales que gestionan.

Así pueden observarse distintos modelos de gestión ensayados con múltiples organismos con distinta jerarquía y composición (Direcciones, Secretarías, Áreas de Coordinación, Ministerios, Agencias).

Las provincias han sido pioneras en la regulación de sus leyes generales de ambiente, en la incorporación de la temática a nivel constitucional y en algunos casos en el uso de instrumentos de gestión. (Como por ej. Córdoba con el uso de la evaluación de impacto ambiental desde 1990).

Ante la reforma constitucional y la sanción de las leyes de presupuestos mínimos, les comprenden por una parte el deber de "proveer" que comentáramos en el art. 41, también en el tercer párrafo cuando distribuye las competencias, las provincias les corresponde complementar las normas de presupuestos mínimos.

El dictado de las leyes de presupuestos mínimos impone un rol a las provincias, en muchos casos avanza sobre las competencias propias de las provincias de acuerdo al art. 124 in fine, situación destacada en la doctrina y en discusión en muchas de las normas.

La gestión ambiental provincial es uno de los protagonistas centrales en la tarea ambiental; con el dominio de los recursos naturales, el deber de su uso racional, el respeto a la diversidad biológica, natural y cultural le dan un marco complejo a las provincias en su obligación de proveer.

Los municipios, constituyen la autoridad local más próxima a los problemas, tienen competencia en su ámbito jurisdiccional en materia ambiental y en muchos casos un rol protagónico por la inmediatez para enfrentar y abordar los problemas.

En el caso de la provincia de Córdoba, donde los municipios cuentan con una autonomía importante, las materias vinculadas al ambiente están delegadas a los municipios en la propia Constitución de la provincia (art. 186 inc. 7).

Las provincias hoy se encuentran con una enorme tarea por delante para dar cumplimiento al mandato constitucional y adecuarse a las nuevas leyes de presupuestos mínimos. 


\section{EL ROL DEL CONSEJO FEDERAL DE MEDIO AMBIENTE (COFEMA)}

Las leyes de presupuestos mínimos han delegado en el Consejo Federal de Medio Ambiente (COFEMA) muchos aspectos a consensuar en forma previa, que van a permitir la implementación de las leyes de presupuestos mínimos (ejemplo de ello, la determinación de los niveles de riesgo en la Ley 25.612).

Si se analizan todas las leyes, a excepción de la referida a gestión ambiental de aguas (Ley Nacional 25688) que es la única que no remite a discusión o consenso en el COFEMA, existe una permanente remisión al COFEMA para el acuerdo, consenso y definición de muchos de los temas.

El desarrollo del COFEMA desde su creación en 1990, ha conformado una historia de acuerdos y disensos de los representantes de las provincias y regiones otorgando a la dinámica de discusión de la política ambiental una particular característica.

Los Consejos Federales en sí mismos tienen una vocación y un rol trascendente en la definición de las políticas relacionadas al tema para el cual fueron creados, como en el caso que nos ocupa: ambiente.

La diversidad y extensión de nuestro territorio, como las características geográficas y climáticas han conformado regiones con características diversas y problemáticas comunes que comparten algunas provincias y otras no.

Es evidente que las administraciones provinciales comparten un conjunto de problemas comunes sobre los cuales pueden intercambiar experiencias, coordinar políticas y reflexionar acerca de sus problemas en distintos ámbitos como los que han dado origen a los "Consejos Federales".

Existen, por otra parte, áreas en las que distintas administraciones necesariamente confluyen en sus competencias y jurisdicciones como son algunos temas interjurisdiccionales donde se ven involucradas dos o más provincias y la Nación en su rol de coordinador de políticas, y que han motivado en muchos casos la conformación de ámbitos para el acuerdo, el consenso, la resolución de conflictos donde los distintos actores involucrados compartan una instancia en común.

Es por tanto, que en uso de sus propias atribuciones las provincias han generado la creación de Consejos Federales como el ámbito propicio para la discusión, el debate, la coordinación política, la generación de acuerdos y consensos para la ejecución de políticas sobre ciertas temáticas determinadas.

Los Consejos Federales conforman un ámbito donde todos los actores, gestores de una política en común, se sientan a la mesa del dialogo en igualdad de condiciones. Los representantes de las administraciones provinciales, el representante de la administración nacional y también el de la Ciudad Autónoma de Buenos Aires.

El Consejo Federal de Medio Ambiente (COFEMA), cuya acta constitutiva se realiza en 1990 producto del Pacto Federal Ambiental, se reconoce formalmente en la Ley General del Ambiente No. 25.675.

Los temas que convocan a la creación de un Consejo Federal, tienen la amplitud propia de los ámbitos administrativos que representan y que forman parte de la 
estructura organizacional de las administraciones públicas, tanto nacional como provinciales, que se encuentran convocadas y aunadas por una temática común que deben gestionar.

La vocación federal de ciertas áreas de Gobierno, ha sido el motor para incentivar la conformación de ámbitos de participación y discusión de los problemas compartidos por las distintas administraciones en los distintos ámbitos jurisdiccionales.

Las provincias argentinas han tenido un constante protagonismo en los Consejos Federales en las distintas temáticas que hoy existen y les han dado jerarquía y relevancia a través de las discusiones, los acuerdos, la políticas concertadas y consensuadas en su seno e informando y difundiendo sus logros, objetivos y problemas.

\section{REFLEXIONES FINALES}

Los espacios institucionales formales representan ricos objetos de investigación en materia ambiental, desde la concepción del Estado en sus diferentes expresiones como espacios centrales e ineludibles en las tareas tendientes a la regulación, prohibición y sanción de actividades degradantes del medio ambiente.

En el marco de las políticas públicas que se desarrollan en materia ambiental, se hace referencia a la necesidad de implementar y contar con espacios de articulación y coordinación adecuados, ya que en numerosas ocasiones el desarrollo de una política determinada se encuentra con los obstáculos propios de una administración compartimentada. Situación que puede observarse en las distintas temáticas, no es sólo observable en el tema ambiental.

Algunos modelos de gestión ambiental diseñados han concebido la gestión como una instancia de coordinación, bajo diferentes formatos (comisión interministerial, coordinador de políticas, entre otros).

Las críticas a las gestiones organizadas como comisiones o áreas de coordinación, se han centrado en la desconfianza que generan en las áreas tradicionales, que en muchos casos quedan marginados en los gabinetes y no logran insertar la dimensión ambiental. En el actual diseño organizacional del Gobierno nacional, se pueden observar espacios concretos donde el objetivo central es la coordinación con el resto de las áreas vinculadas a las temáticas que son objeto de ejecución de una política determinada.

Cada administración distribuye en un número de ministerios determinados la ejecución de la política, con su respectiva organización, y a su vez, puede insertar o no en el diseño las instancias de coordinación o articulación de tipo horizontal.

En el diseño de la administración actual, que rige desde 2003, se pueden observar múltiples espacios destinados a generar la articulación entre los distintos sectores vinculados de la administración. 
Se establecen, específicamente en los objetivos de estas áreas, con el uso del término "coordinar" las políticas con los sectores pertinentes y en algunos casos se establece con las áreas que va a coordinar en determinadas temáticas. Es precisa la definición en la organización de los espacios asignados para realizar las tareas de coordinación, y en el mapa general de la organización del Gobierno nacional puede visualizarse claramente en diversos lugares.

Se ha remarcado la necesidad de establecer formas de coordinación entre los distintos sectores de la administración en la organización estatal, con el objeto de optimizar las actividades, lograr eficiencia y superar cuestiones meramente burocráticas.

Las tareas de coordinación y formulación de políticas no se tenían en cuenta en el marco de las políticas de la economía de mercado, ya que se suponía la no necesidad de la regulación coordinada del Estado en espacios librados a las reglas de mercado. Sin embargo, en los últimos tiempos han vuelto a adquirir entidad conceptual en los espacios de gestión pública.

Existen instancias de coordinación de políticas en el marco de la Administración Pública Nacional, están organizadas, pero es necesario analizar los alcances y oportunidades que se observan.

Hemos destacado en otros trabajos (Juliá \& del Campo, 2007) y reiteramos algunos puntos que se deben considerar y reafirmar en materia de coordinación en la gestión:

- En la formulación de políticas macroeconómicas sería importante la consideración de la presión que se ejerce y se van a ejercer sobre los recursos naturales no renovables como una variable a incluir.

- En los programas que se han consolidado a través de los compromisos internacionales asumidos por Argentina lograr la implementación efectiva de dichos programas.

- En algunos espacios específicos se observa la idea de coordinar en forma previa como es el caso de la política ambiental íntimamente vinculada al sector hídrico; es un buen indicio en la verdadera implementación de la instancia coordinada de definición de las políticas públicas en esta área.

- No se puede coordinar ni integrar políticas si no hay información acerca de las políticas, de la gestión, y por tanto participación de los sectores involucrados en la decisión.

- Cada área debe conocer con quienes coordinar, los mecanismos creados y vigentes y cuáles son los funcionarios que constituyen el nexo institucional con quien deben sentarse a coordinar la política en común.

- Es necesario capacitar para coordinar desde la propia administración a los técnicos y funcionarios. Muchos no tienen experiencias en trabajos interinstitucionales e interjurisdiccionales, para lo cual la administración necesita capacitar e incorporar la complejidad en la estructuras de las decisiones de cada área de tal manera que puedan percibir la idea de la política coordinada e integral. 
Cuando hacemos referencia a la coordinación de políticas incluimos la coordinación política, institucional y administrativa, ya que debe operativizarse en sus tres componentes.

En la implementación institucional y en la coordinación legal no deben dejarse espacios vacíos que den lugar a la inseguridad jurídica. En la distribución de funciones en la organización suelen quedar aspectos vinculados a dos sectores o no estar claramente delimitados, son áreas de interacción entre distintos organismos que deben articularse en forma clara.

En materia administrativa nada impide la actuación conjunta de organismos, en el marco de sus atribuciones se pueden llevar a cabo una serie de actividades tendientes a la prevención y tareas de inspección, control y fiscalización.

La coordinación interjurisdiccional supone un esfuerzo mayor, ya que la relación entre los distintos niveles gubernamentales es de mayor complejidad existiendo numerosas formas e instancias para su implementación.

En líneas generales puede afirmarse que las instancias formales existen, lo que no implica que la coordinación se realice. Creo que la participación ciudadana y la coordinación de políticas tienen en común el haberse construido los canales formales y carecer aún del adecuado ejercicio de los mismos.

Hay discusiones de políticas que deben realizarse en las instancias específicas y en los espacios creados para ello como son los Consejos Federales. Las áreas de coordinación y el ejercicio de la discusión, el respeto a la diversidad, el disenso, nos va a permitir implementar estas ideas en la práctica de la administración.

El ejercicio de la coordinación y articulación institucional constituye un desafío a intentar para la implementación de las políticas ambientales en el sector público, un gran paso en la consolidación de la gestión ambiental.

\section{BIBLIOGRAFÍA}

- Brañes, R. (2001). Informe sobre desarrollo del Derecho Ambiental Latinoamericano. PNUMA, oficina regional para América Latina, México. - Cancino, M.A. (2003). Reflexiones en torno a los mecanismos de aplicación y cumplimiento de la normativa ambiental en México. $1^{\circ}$ Conferencia Internacional sobre Aplicación y Cumplimiento de la Normativa Ambiental en América Latina FARN-, Buenos Aires. - Etchichury, H.J. (2006). Tres enfoques sobre el derecho al medio ambiente. Córdoba: $\quad$ Lerner. - Juliá, M.S. y del Campo, M.C. (2007). ¿La coordinación interinstitucional: un factor condicionante para la gobernabilidad del agua? Trabajo presentado en el II Congreso Pampeano del Agua, General Pico, La Pampa. - Juliá, M.S.; del Campo, C. y Foa Torres, J. (2009). La institucionalización ambiental en Argentina. Córdoba: Lerner. - Ozlak, O. (1980). Políticas Públicas y Regímenes Políticos: Reflexiones a partir de algunas experiencias Latinoamericanas. Documento de Estudios CEDES, Vol. 3 No. 2. Buenos Aires. p. 4. 
1. Doctora en Derecho y Ciencias Sociales de la Universidad Nacional de Córdoba, Argentina. Investigadora del Centro de Investigaciones Jurídicas y Sociales de la Facultad de Derecho de la UNC, docente en Derecho Ambiental en diferentes Maestría en Universidades Nacional y Tecnológica en Córdoba, Catamarca, Santa Fé de Argentina.

2. En amplitud se puede consultar Juliá, del Campo y Foa (2009).

3. "Art. 41 - Todos los habitantes gozan el derecho a un ambiente sano, equilibrado, apto para el desarrollo humano y para que las actividades productivas satisfagan las necesidades presentes sin comprometer las de las generaciones futuras y tienen el deber de preservarlo. El daño ambiental generará la obligación de recomponer, según lo establezca la ley".

4. "ARTÍCULO $2^{\circ}$ - La política ambiental nacional deberá cumplir los siguientes objetivos:

a. Asegurar la preservación, conservación, recuperación y mejoramiento de la calidad de los recursos ambientales, tanto naturales como culturales, en la realización de las diferentes actividades antropicas; b. Promover el mejoramiento de la calidad de vida de las generaciones presentes y futuras, en forma prioritaria; c. Fomentar la participación social en los procesos de toma de decisión; d. Promover el uso racional y sustentable de los recursos naturales; e. Mantener el equilibrio y dinámica de los sistemas ecológicos; f. Asegurar la conservación de la diversidad biológica; g. Prevenir los efectos nocivos o peligrosos que las actividades antropicas generan sobre el ambiente para posibilitar la sustentabilidad ecológica, económica $y$ social del desarrollo; h. Promover cambios en los valores y conductas sociales que posibiliten el desarrollo sustentable, a través de una educación ambiental, tanto en el sistema formal como en el no formal; i. Organizar e integrar la información ambiental y asegurar el libre acceso de la población a la lasma; j. Establecer un sistema federal de coordinación interjurisdiccional, para la implementación de políticas ambientales de escala nacional y regional; k. Establecer procedimientos y mecanismos adecuados para la minimización de riesgos ambientales, para la prevención y mitigación de emergencias ambientales y para la recomposición de los daños causados por la contaminación ambiental".

5. http://ambiente.gov.arl (9/10/2007). 